\title{
Proteogenomic Study beyond Chromosome 9: New Insight into Expressed Variant Proteome and Transcriptome
}

\section{in Human Lung Adenocarcinoma Tissues}

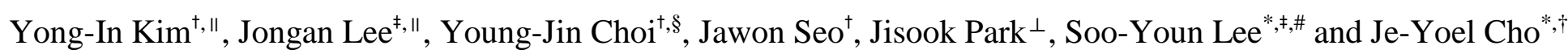

${ }^{\dagger}$ Department of Biochemistry, BK21 PLUS Program for Creative Veterinary Science Research and Research Institute for Veterinary Science, College of Veterinary Medicine, Seoul National University, Seoul 08826, South Korea; ₹Department of Laboratory Medicine \& Genetics, Samsung Medical Center, Sungkyunkwan University School of Medicine, Seoul 06351, South Korea; ${ }^{\S}$ ProtAnBio, Seoul 08826, South Korea; ${ }^{\perp}$ Samsung Biomedical Research Institute, Samsung Medical Center, Sungkyunkwan University School of Medicine, Seoul 06351, South Korea; \#Department of Clinical Pharmacology \& Therapeutics, Samsung Medical Center, Seoul 06351, South Korea

"These authors contributed equally.

*Co-corresponding author

\section{TABLE OF CONTENTS}

Supplementary data 1 . Representative spectra of the missing protein

Supplementary data 2. Representative spectra of the nonsynonymous variant

Supplementary data 3. Representative spectra of IGKV6D-21

Supplementary data 4. Detection of IGKV6D-21 expression at the transcript level
Page S-2

Page S-6

Page S-10

Page S-12 
Supplementary data 1 . Representative spectra of the missing protein

\section{SLC35A4}

TLRDYLQLLR

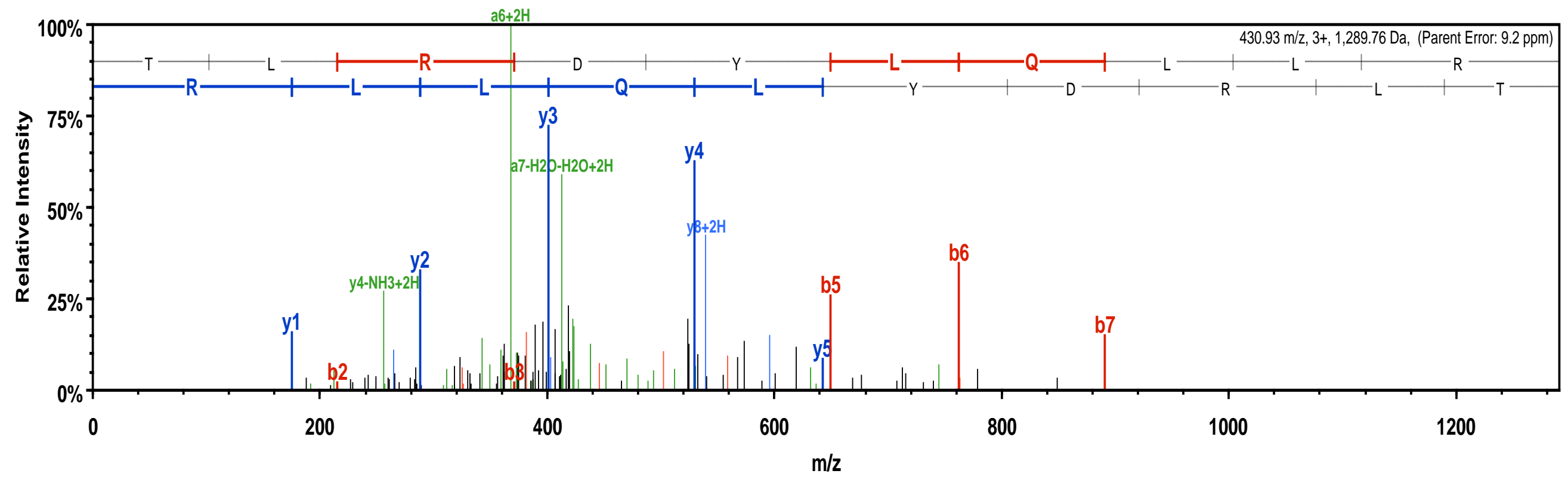


SLC35A4

GFLAGYVVAK

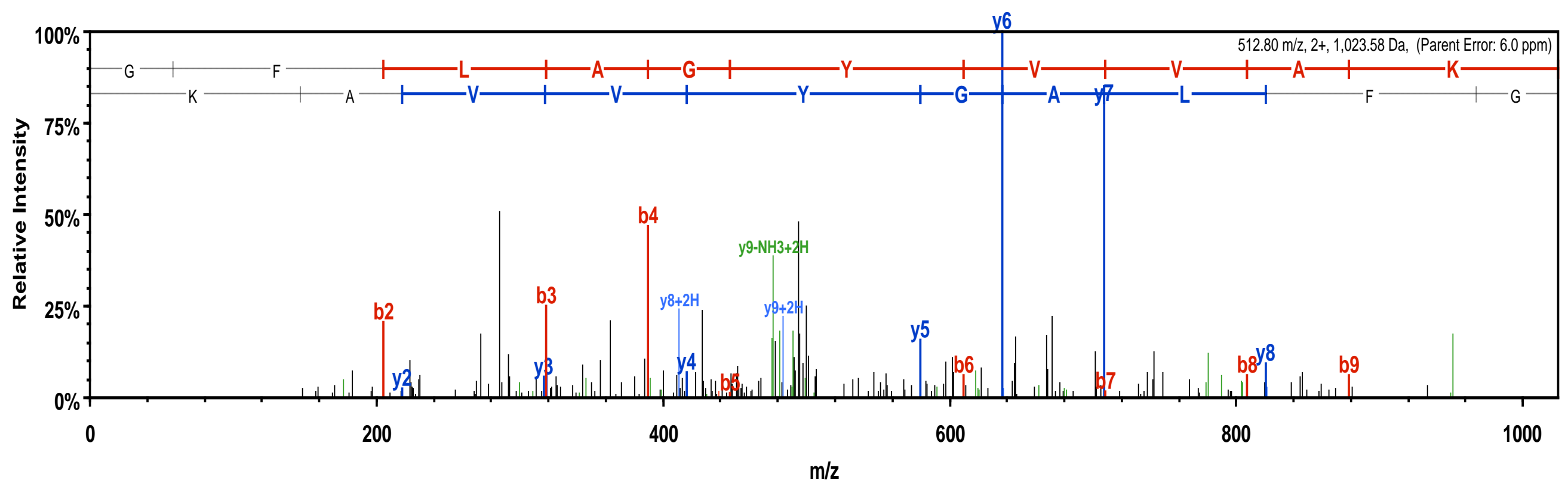


CCDC168

KLLGSLSGSLTTVFH

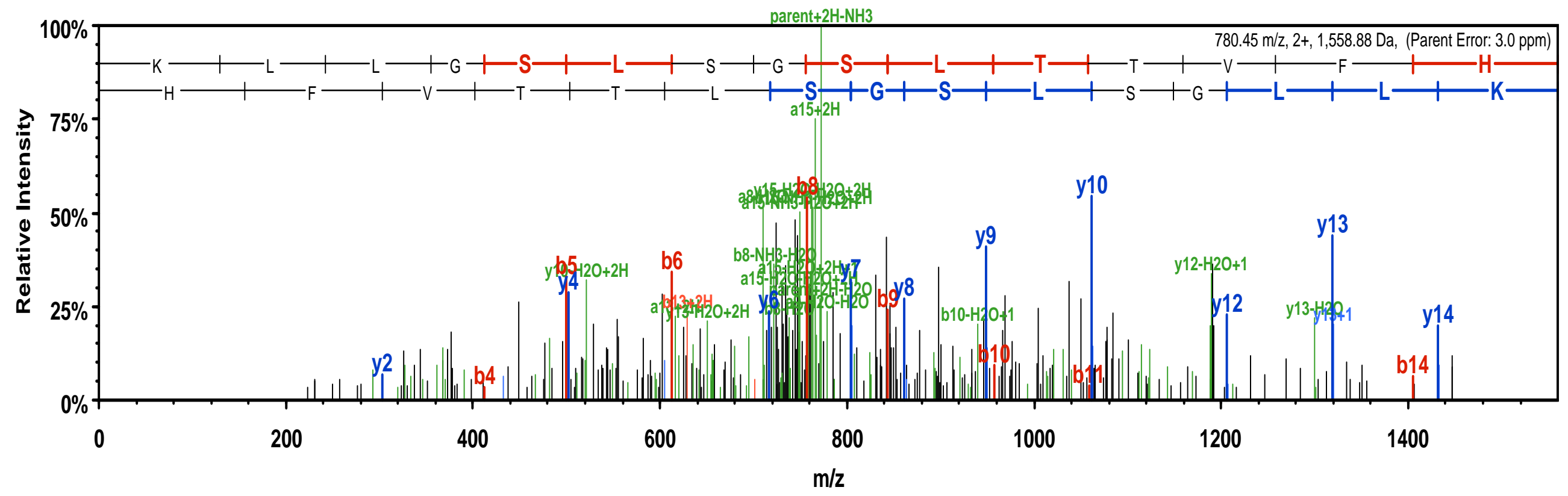


CCDC168

IPLENVYK

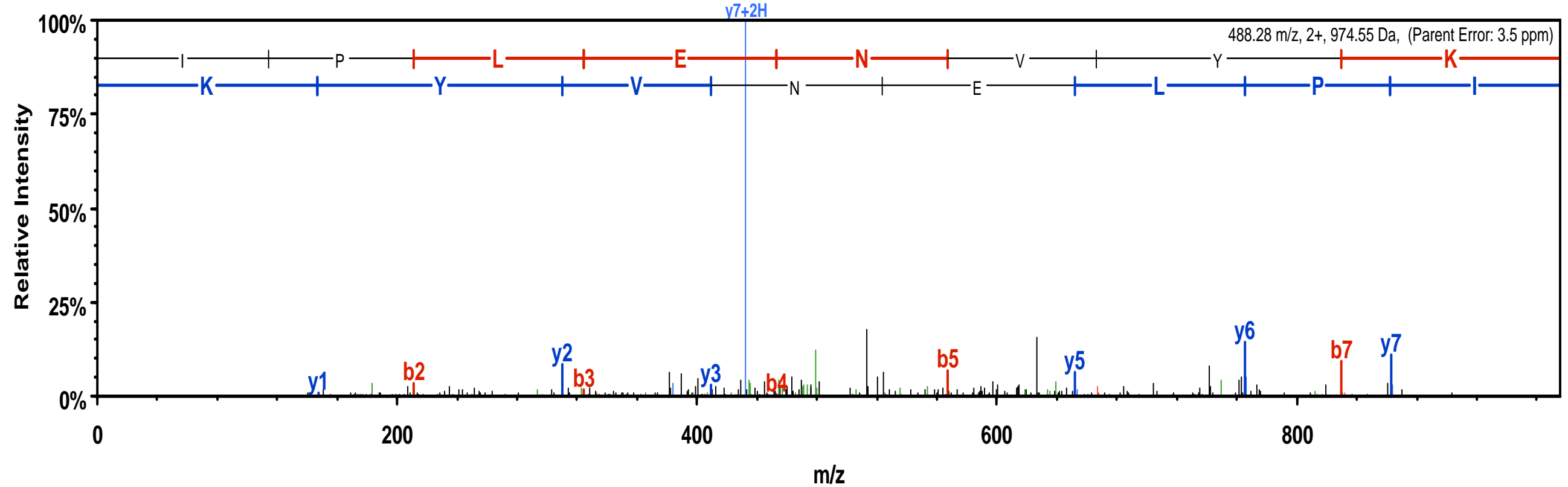


Supplementary data 2. Representative spectra of the nonsynonymous variant

$\operatorname{HDLBP}(\mathrm{S} 61 \mathrm{~A})$

AACLESAQEPAGGAWGNK

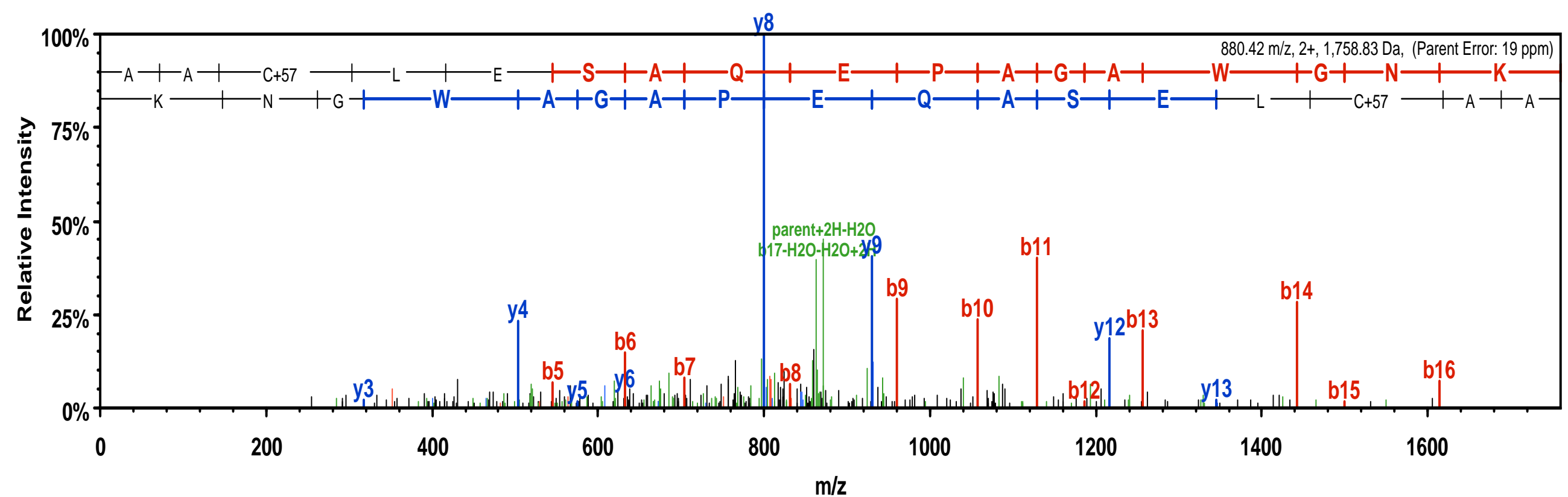


LTF(E535D)

DVTVLQNTDGNNNDAWAK

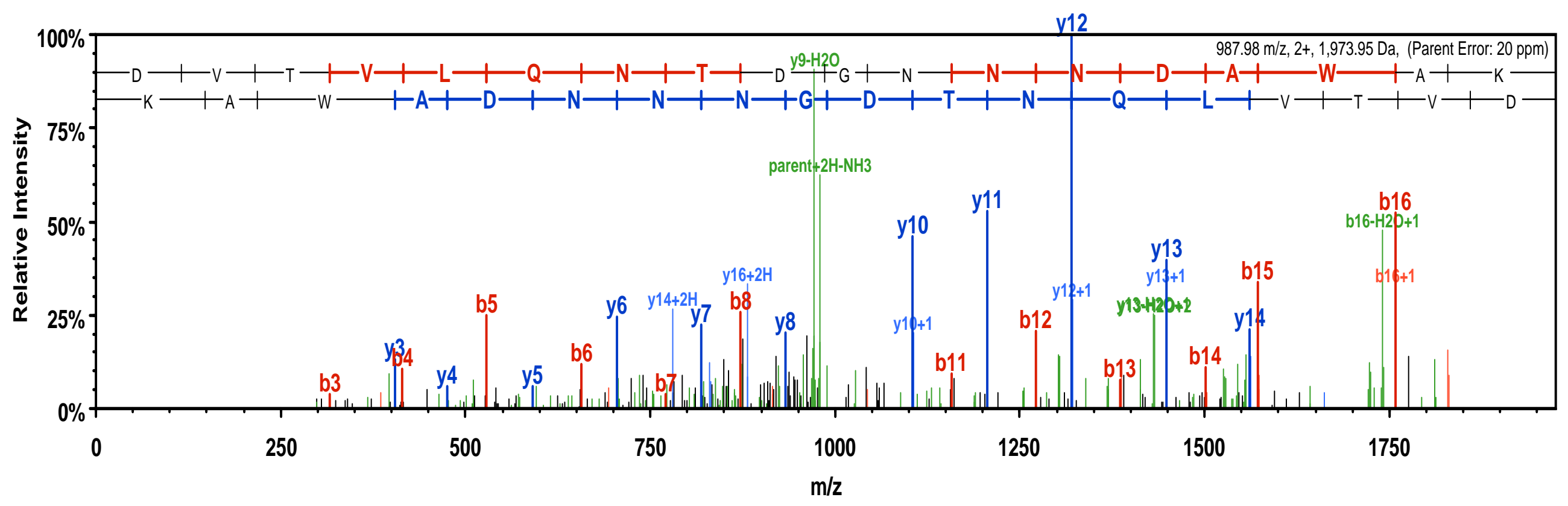


TF(P589S)

SVEEYANCHLAR

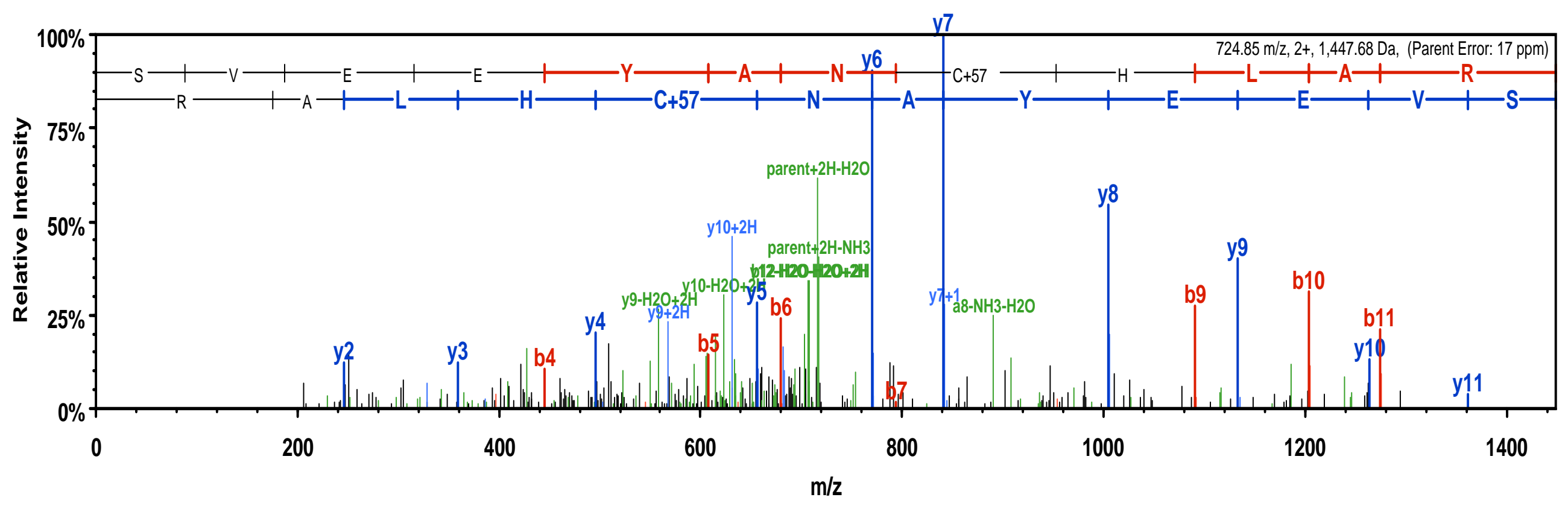


HBD(A23E)

VNVDEVGGEALGRL

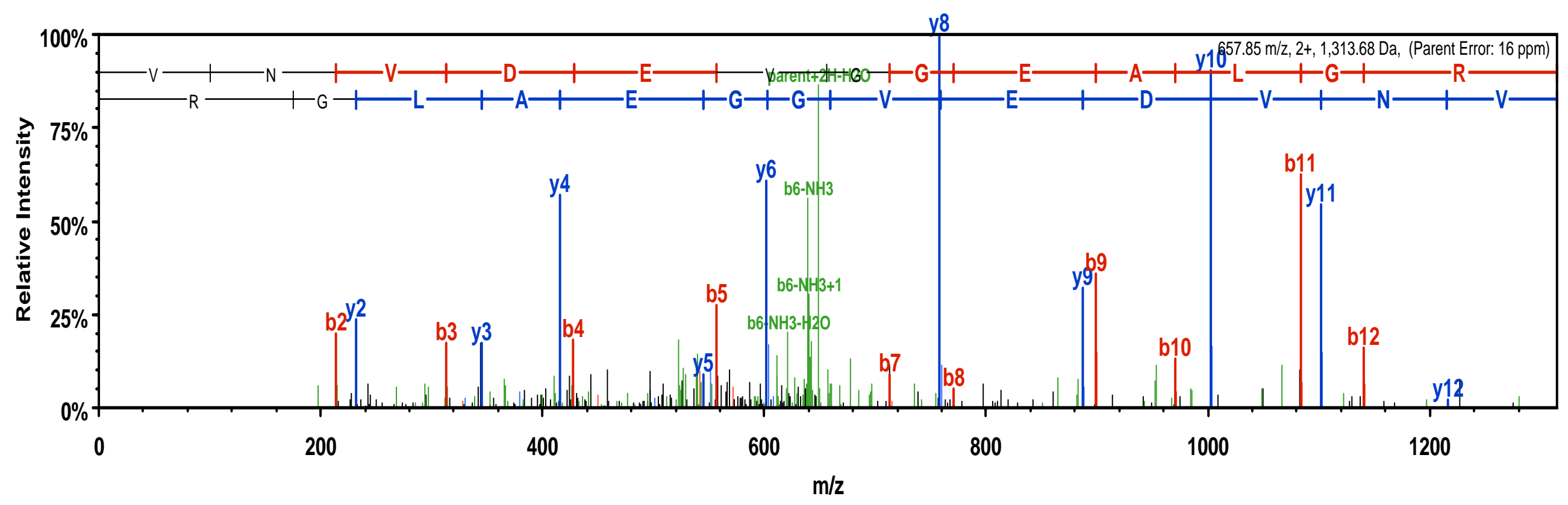


Supplementary data 3. Representative spectra of IGKV6D-21

\section{EIVLTQSPDFQSVTPK}

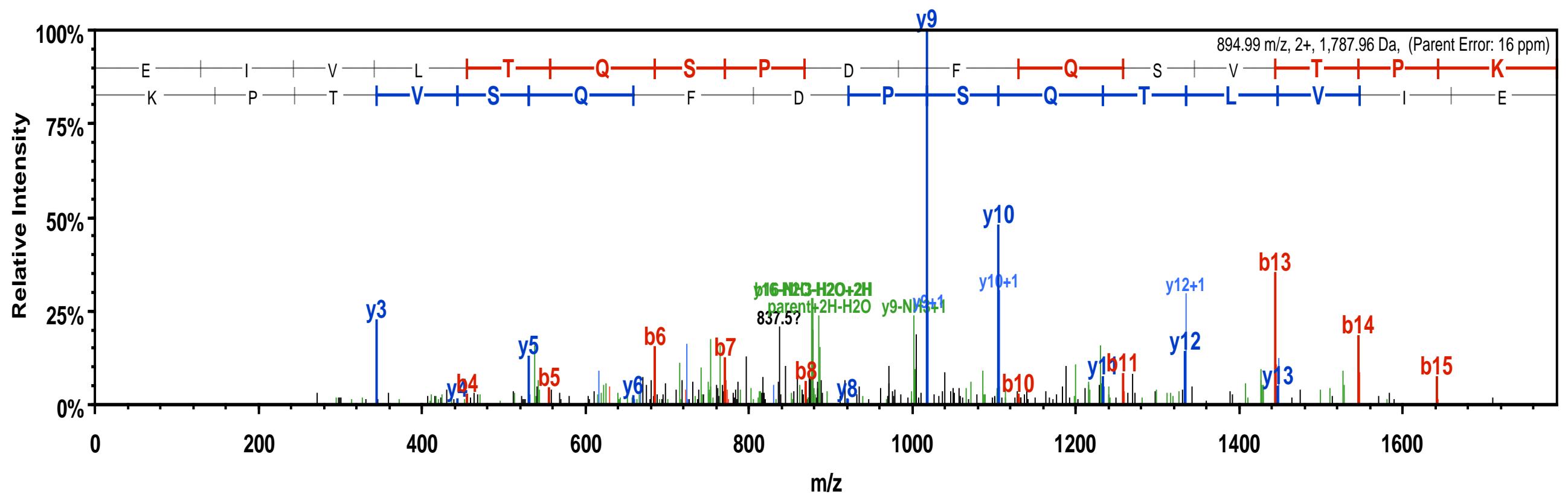


YASQSISGVPSR

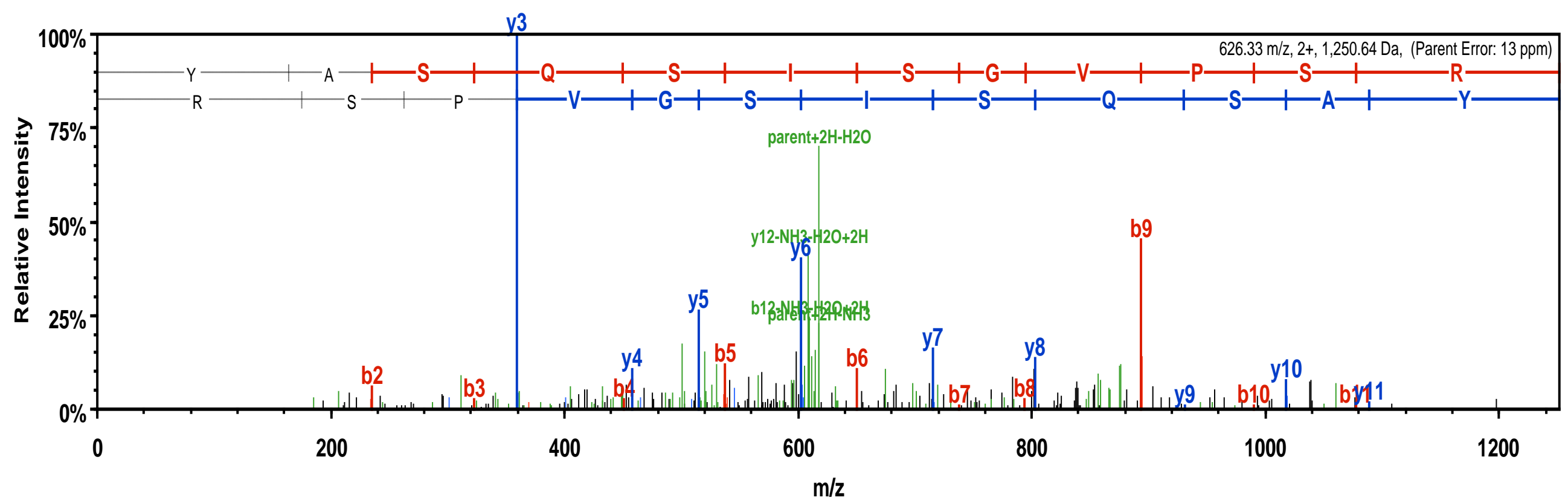


Supplementary data 4. Detection of IGKV6D-21 expression at the transcript level

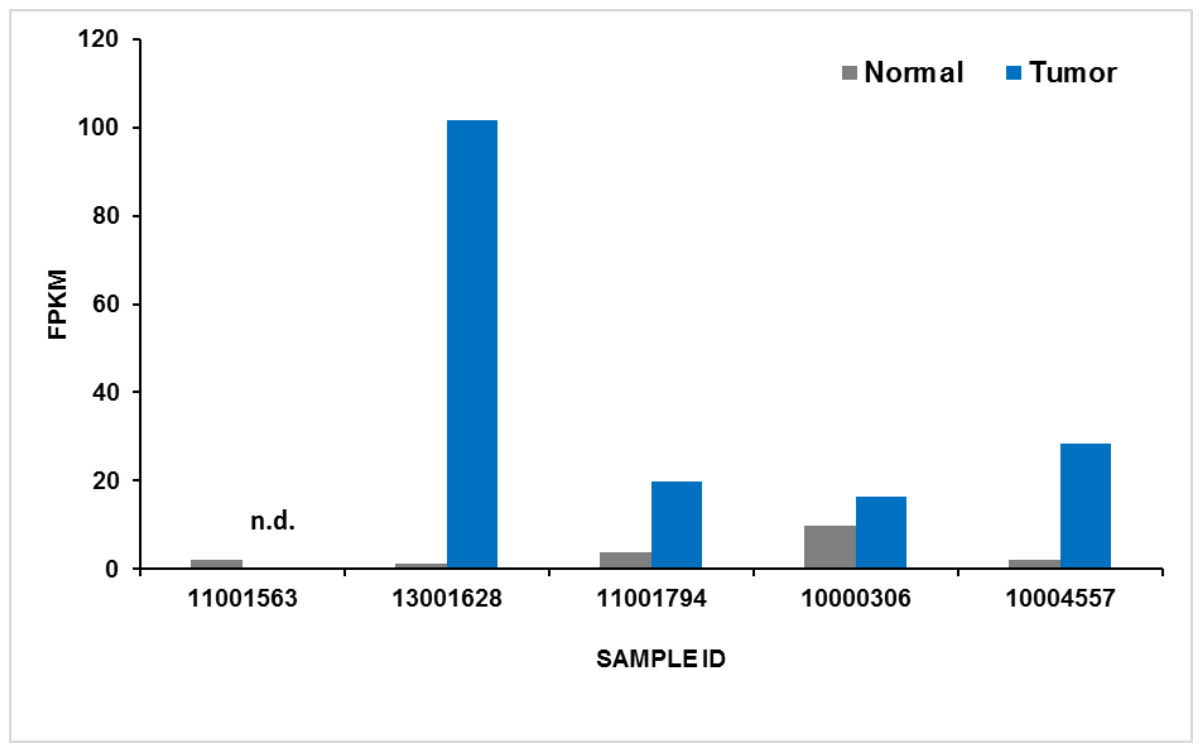

